\title{
Mutations in NGLY1 cause an inherited disorder of the endoplasmic reticulum-associated degradation pathway
}

\author{
Gregory M. Enns, MB, ChB ${ }^{1}$, Vandana Shashi, MD, MBBS², Matthew Bainbridge, $\mathrm{PhD}^{3}$, \\ Michael J. Gambello, MD, PhD , Farah R. Zahir, PhD ${ }^{5}$, Thomas Bast, MD, Rebecca Crimian, MS², \\ Kelly Schoch, MS², Julia Platt, MS', Rachel Cox, MS', Jonathan A. Bernstein, MD, PhD', \\ Mena Scavina, $\mathrm{DO}^{7}$, Rhonda S. Walter, $\mathrm{MD}^{8}$, Audrey Bibb, MS4, Melanie Jones, $\mathrm{PhD}^{4}$, \\ Madhuri Hegde, PhD ${ }^{4}$, Brett H. Graham, MD, PhD ${ }^{3}$, Anna C. Need, PhD ${ }^{9}$, Angelica Oviedo, MD ${ }^{10}$, \\ Christian P. Schaaf, MD, PhD ${ }^{3,11}$, Sean Boyle, PhD ${ }^{12}$, Atul J. Butte, MD, PhD ${ }^{12}$, Rong Chen, PhD ${ }^{12}$, \\ Michael J. Clark, PhD ${ }^{12}$, Rajini Haraksingh, PhD ${ }^{12}$, Tina M. Cowan, PhD ${ }^{13}$, \\ FORGE Canada Consortium, Ping He, MD, PhD ${ }^{14}$, Sylvie Langlois, MD ${ }^{5}$, Huda Y. Zoghbi, MD ${ }^{3,11,15}$, \\ Michael Snyder, PhD ${ }^{12}$, Richard A. Gibbs, PhD ${ }^{3,16}$, Hudson H. Freeze, PhD ${ }^{14}$ and David B. Goldstein, PhD ${ }^{17,18}$
}

\begin{abstract}
Purpose: The endoplasmic reticulum-associated degradation pathway is responsible for the translocation of misfolded proteins across the endoplasmic reticulum membrane into the cytosol for subsequent degradation by the proteasome. To define the phenotype associated with a novel inherited disorder of cytosolic endoplasmic reticulumassociated degradation pathway dysfunction, we studied a series of eight patients with deficiency of $\mathrm{N}$-glycanase 1.
\end{abstract}

Methods: Whole-genome, whole-exome, or standard Sanger sequencing techniques were employed. Retrospective chart reviews were performed in order to obtain clinical data.

Results: All patients had global developmental delay, a movement disorder, and hypotonia. Other common findings included hypolacrima or alacrima (7/8), elevated liver transaminases (6/7), microcephaly (6/8), diminished reflexes (6/8), hepatocyte cytoplasmic stor- age material or vacuolization (5/6), and seizures (4/8). The nonsense mutation c.1201A $>\mathrm{T}$ (p.R401X) was the most common deleterious allele.

Conclusion: NGLY1 deficiency is a novel autosomal recessive disorder of the endoplasmic reticulum-associated degradation pathway associated with neurological dysfunction, abnormal tear production, and liver disease. The majority of patients detected to date carry a specific nonsense mutation that appears to be associated with severe disease. The phenotypic spectrum is likely to enlarge as cases with a broader range of mutations are detected.

Genet Med advance online publication 20 March 2014

Key Words: alacrima; choreoathetosis; liver disease; NGLY1; seizures

\section{INTRODUCTION}

The enzyme N-glycanase 1 (NGLY1), also known as peptide:Nglycanase (PNGase, EC 3.5.1.52), catalyzes protein deglycosylation by cleaving the $\beta$-aspartyl glycosylamine bond of $\mathrm{N}$-linked glycoproteins with the subsequent release of intact $\mathrm{N}$-glycan species. ${ }^{1}$ NGLY1 activity was first described in almond seeds ${ }^{1}$ but is ubiquitously present across a wide range of species, including bacteria, ${ }^{2}$ yeast, ${ }^{3-5}$ amoeba, ${ }^{6}$ fish, ${ }^{7}$ and mammals. ${ }^{8,9}$ Evidence suggests that NGLY1 participates as a key cytoplasmic component of the endoplasmic reticulum-associated degradation (ERAD) machinery along with the AAA ATPase complex p97.10,11 The ERAD pathway is a mechanism for identifying and degrading misfolded glycoproteins. $\mathrm{N}$-glycans that are high in mannose content act as quality control tags for proteins in the early stages of the secretory pathway. ${ }^{12,13}$ Misfolded glycoproteins are detected by ER luminal lectins and are then translocated to the cytosol via the ERAD machinery to be subsequently degraded by cytosolic enzymes including NGLY $1 .{ }^{14}$

To date, a single patient with NGLY1 deficiency has been reported as part of a whole-exome sequencing (WES) study

\footnotetext{
The first three authors contributed equally to this study.

${ }^{1}$ Department of Pediatrics, Division of Medical Genetics, Lucile Packard Children's Hospital, Stanford University, Stanford, California, USA; ${ }^{2}$ Department of Pediatrics, Division of Medical Genetics, Duke University, Durham, North Carolina, USA; ${ }^{3}$ Department of Molecular and Human Genetics, Baylor College of Medicine, Houston, Texas, USA; ${ }^{4}$ Department of Human Genetics, Division of Medical Genetics, Emory University School of Medicine, Atlanta, Georgia, USA; ${ }^{5}$ Department of Medical Genetics, University of British Columbia, Vancouver, British Columbia, Canada; ${ }^{6}$ Epilepsy Centre Kork, Kehl, Germany; 7 Division of Pediatric Neurology, Department of Pediatrics, Nemours/Alfred I. duPont Hospital for Children, Wilmington, Delaware, USA; ${ }^{8}$ Division of Developmental Medicine, Department of Pediatrics, Nemours/Alfred I. duPont Hospital for Children, Wilmington, Delaware, USA; ${ }^{9}$ Department of Medicine, Imperial College, London, UK; ${ }^{10}$ Department of Pathology and Laboratory Medicine, Dalhousie University, Halifax, Nova Scotia, Canada; ${ }^{11}$ Jan and Dan Duncan Neurological Research Institute, Texas Children's Hospital, Houston, Texas, USA; ${ }^{12}$ Department of Genetics, Stanford University, Stanford, California, USA; ${ }^{13}$ Department of Pathology, Stanford University, Stanford, California, USA; ${ }^{14}$ Sanford-Burnham Medical Research Institute, La Jolla, California, USA; ${ }^{15}$ Howard Hughes Medical Institute, Baylor College of Medicine, Houston, Texas, USA; ${ }^{16}$ Human Genome Sequencing Center, Baylor College of Medicine, Houston, Texas, USA; ${ }^{17}$ Center for Human Genome Variation and Department of Medicine, Duke University School of Medicine, Durham, North Carolina, USA; ${ }^{18}$ Department of Molecular Genetics and Microbiology, Duke University School of Medicine, Durham, North Carolina, USA. Correspondence: Gregory M. Enns (greg.enns@stanford.edu)
} 
focusing on the utility of this technology to detect the underlying genetic etiology of disorders affecting patients with previously undiagnosed or unresolved genetic conditions. ${ }^{15}$ Need et al. ${ }^{15}$ described a 3-year-old boy with compound heterozygous inactivating mutations in NGLY1 and suggested that this could be a new disorder. The initial patient had a clinical phenotype suggestive of a congenital disorder of glycosylation (CDG), although repeated transferrin isoelectric focusing and N-glycan analyses were normal. Liver biopsy showed accumulation of an amorphous unidentified substance throughout the cytoplasm-a finding likely consistent with NGLY1 dysfunction-which would be expected to result in abnormal accumulation of misfolded glycoproteins because of impaired cytosolic degradation..$^{15}$ We now report clinical and molecular findings present in seven newly diagnosed patients with mutations in NGLY1, as well as further details related to the original case. These observations confirm NGLY1 deficiency as an inherited disorder associated with the ERAD process and document its clinical presentation.

\section{MATERIALS AND METHODS}

The Stanford Institutional Review Board approved this study. Clinical data were abstracted from the medical records by retrospective chart review. Further data were collected by direct interaction with the families of participating subjects. Ascertainment occurred by patient families, treating physicians, or both directly contacting the primary or senior authors following the publication of the original article describing a case of NGLY1 deficiency. ${ }^{15}$ An Internet blog started by the father of the initial patient was instrumental in connecting NGLY1-deficient patient families with physicians and scientists studying this novel disorder (see accompanying Commentary by Might and Wilsey in this issue).

Exome sequencing was performed in Patient 1 (Duke University), Patients 5 and 6 (University of British Columbia), and their parents using the Illumina HiSeq2000 platform and the Agilent SureSelect Human All Exon 50 Mb Kit. Patient 3 was evaluated independently at both Stanford University and Baylor College of Medicine. For patient 3, samples were sequenced using Illumina HiSeq2000 and Complete Genomics platforms (Stanford University) and in addition by DNA capture (Baylor College of Medicine) using a commercially developed reagent (VCRome2) and an Illumina HiSeq2000 (average coverage of 80x with $>90 \%$ of targeted bases at $20 \mathrm{x}$ coverage or higher). WES was performed on a clinical basis in three patients (Patient 2 (Baylor College of Medicine Whole Genome Laboratory, Houston, TX) and Patients 7 and 8 (Emory Genetics Laboratory, Atlanta, GA)). For all patients, variants were confirmed by Sanger sequencing (Supplementary Table S1 online). Sanger sequencing of NGLY1 was performed in Patient 4 at Duke University, and results were confirmed by a clinical laboratory (GeneDx, Gaithersburg, MD).

\section{RESULTS}

\section{Case reports}

The clinical and molecular findings demonstrate clear clinical similarities of patients with NYGL1 deficiency, although some variation in overall severity was observed, with the most common mutation being associated with more severe outcomes (Table 1). All patients had global developmental delay, a movement disorder, and hypotonia. Other common findings included hypolacrima or alacrima (7/8), electroencephalography (EEG) abnormalities (7/8), elevated liver transaminases (6/7), microcephaly (6/8), abnormal brain imaging (6/8), diminished reflexes (6/8), hepatocyte cytoplasmic storage material or vacuolization (5/6), seizures (4/8), and abnormal nerve conduction (3/3) (Figure 1). Although all patients had cognitive, motor, and speech impairment, developmental regression was noted only in Patients 5 and 8. Patients did not have significant synthetic dysfunction or a coagulopathy, despite the elevations in liver transaminases. Two of the patients died prematurely, at 9 months and 5 years of age, respectively. Pedigrees for all patients are shown in Figure 2. Short clinical summaries are provided below. Further details may be found in the Supplementary Materials and Methods online.

Patient 1, a now 5-year-old boy, presented in the neonatal period with involuntary movements, including athetosis involving the trunk and extremities and constant lip smacking and pursing while awake. Global developmental delay; hypotonia; intractable multifocal epilepsy, consisting of myoclonic seizures, drop attacks, and staring or tonic episodes; and liver disease were present in infancy. He has cortical vision impairment and congenital alacrima, and corneal ulcerations with scarring were noted at the age of 4 years. Now, at 5 years of age, the movement disorder has not abated and he has central hypotonia and global developmental delay.

Patient 2, a now 20-year-old female, was born at 39 weeks of gestation via Cesarean section because of intrauterine growth restriction (IUGR) and an abnormal-appearing placenta on ultrasonography. At 4 months of age, hypotonia, developmental delay, and elevated liver transaminases were noted. At $\sim 4$ years of age, a slight intention tremor and frequent involuntary movements of her neck, hands, and arm were observed. At 5 years of age, she was noted to have ocular apraxia, distal tapering of hands and feet, and diminished deep tendon reflexes. She has cortical vision impairment, as well as alacrima and dry eyes that require lubrication, but has not developed corneal scarring. Currently, she has cognitive, motor, and speech impairment and requires total care. She has very little expressive speech and communicates through an electronic speech-generating device. She ambulates with a walker.

Patient 3, a now 4-year-old girl, was born via Cesarean section at term for a nonreassuring fetal heart tracing and was monitored in the neonatal intensive care unit because of poor feeding and lethargy. The pregnancy was complicated by a positive second-trimester screen noting increased risk for Smith-Lemli-Opitz syndrome and trisomy 18 ( $\alpha$-fetoprotein (AFP) 1.63 multiple of the median (MoM), unconjugated estriol (uE3) $0.26 \mathrm{MoM}$, human chorionic gonadotrophin 0.54 MoM, inhibin $1.02 \mathrm{MoM}$ ), but karyotype on amniocentesis was normal. As a neonate, she had hyperbilirubinemia that was treated with phototherapy and was noted to have elevated liver 
Table 1 Clinical and molecular findings in NGLY1 deficiency

\begin{tabular}{|c|c|c|c|c|c|c|c|c|c|}
\hline & Patient 1 & Patient 2 & Patient 3 & Patient 4 & Patient 5 & Patient 6 & Patient 7 & Patient 8 & Totals \\
\hline Age & 5 years & 20 years & 4 years & 2 years & d. 5 years & d. 9 months & 3 years & 16 years & \\
\hline Gender & M & $\mathrm{F}$ & $\mathrm{F}$ & M & M & $\mathrm{F}$ & $\mathrm{F}$ & $\mathrm{F}$ & \\
\hline Ethnicity & Caucasian & Caucasian & Caucasian & Caucasian & Caucasian & Caucasian & Caucasian & Caucasian & \\
\hline $\begin{array}{l}\text { Mutations (maternal/ } \\
\text { paternal allele) }\end{array}$ & $\begin{array}{l}\text { c.C1891del } \\
\text { (p.Q631fs)/ } \\
\text { c.1201A>T } \\
\text { (p.R401X) }\end{array}$ & $\begin{array}{c}\text { c.1370dupG } \\
\text { (p.R458fs)/ } \\
\text { c.1370dupG } \\
\text { (p.R458fs) }\end{array}$ & $\begin{array}{c}\text { c.1205_1207del } \\
\text { (p.402_403del)/ } \\
\text { c.1570C>T } \\
\text { (p.R524X) }\end{array}$ & $\begin{array}{l}\text { c.1201A>T } \\
\text { (p.R401X)/ } \\
\text { c.1201A>T } \\
\text { (p.R401X) }\end{array}$ & $\begin{array}{l}\text { c.1201A>T } \\
(p . R 401 X) / \\
\text { c.1201A>T } \\
\text { (p.R401X) }\end{array}$ & $\begin{array}{l}\text { c.1201A>T } \\
\text { (p.R401X)/ } \\
\text { c.1201A>T } \\
\text { (p.R401X) }\end{array}$ & $\begin{array}{l}\text { c. } 1201 \mathrm{~A}>\mathrm{Y} \\
(\mathrm{p} . \mathrm{R} 401 \mathrm{X}) / \\
\text { c.1201A>T } \\
(p . R 401 \mathrm{X})\end{array}$ & $\begin{array}{c}\text { c1201A>T } \\
(p . R 401 X) / \\
\text { c.1201A>T } \\
\text { (p.R401X) }\end{array}$ & \\
\hline
\end{tabular}

\begin{tabular}{|c|c|c|c|c|c|c|c|c|c|}
\hline IUGR & - & + & - & + & + & + & - & + & $5 / 8$ \\
\hline $\begin{array}{l}\text { Brain imaging } \\
\text { abnormalities }\end{array}$ & $t^{a}$ & $-{ }^{\mathrm{b}}$ & $+^{c}$ & $t^{d}$ & $+^{\mathrm{e}}$ & $+^{f}$ & - & $+^{9}$ & $6 / 8$ \\
\hline $\begin{array}{l}\text { Global developmental } \\
\text { delay }\end{array}$ & + & + & + & + & + & + & + & + & $8 / 8$ \\
\hline Microcephaly ${ }^{h}$ & - & + & + & - & + & + & + & + & $6 / 8$ \\
\hline Hypotonia & + & + & + & + & + & + & + & + & $8 / 8$ \\
\hline Movement disorder & + & + & + & + & + & + & + & + & $8 / 8$ \\
\hline EEG abnormalities & + & + & + & + & + & + & - & + & $7 / 8$ \\
\hline$\downarrow D T R s$ & + & + & - & + & + & - & + & + & $6 / 8$ \\
\hline Seizures & + & - & - & + & + & - & - & + & $4 / 8$ \\
\hline Ocular apraxia & - & + & + & - & - & - & + & + & $4 / 8$ \\
\hline Alacrima/hypolacrima & + & + & + & + & + & - & + & + & $7 / 8$ \\
\hline $\begin{array}{l}\text { Corneal ulcerations/ } \\
\text { scarring }\end{array}$ & + & + & - & + & - & - & - & + & $4 / 8$ \\
\hline Chalazions & + & - & + & + & - & - & + & - & $4 / 8$ \\
\hline Strabismus & - & - & + & + & - & - & + & + & $4 / 8$ \\
\hline ABR abnormalities & - & - & + & + & - & ND & ND & ND & $2 / 5$ \\
\hline Elevated blood lactate & - & + & + & + & $-{ }^{\mathrm{j}}$ & ND & + & ND & $4 / 6$ \\
\hline Neonatal jaundice & + & - & + & + & - & - & + & - & $4 / 8$ \\
\hline $\begin{array}{l}\text { Elevated liver } \\
\text { transaminases }\end{array}$ & + & + & + & + & + & ND & + & - & $6 / 7$ \\
\hline Elevated AFP & + & - & $-{ }^{k}$ & + & + & ND & ND & ND & $3 / 5$ \\
\hline Liver fibrosis & + & - & - & + & - & - & ND & ND & $2 / 6$ \\
\hline $\begin{array}{l}\text { Liver storage or } \\
\text { vacuolization }\end{array}$ & + & + & + & - & + & $+^{m}$ & ND & ND & $5 / 6$ \\
\hline Constipation & + & + & + & + & + & - & + & + & $7 / 8$ \\
\hline Dysmorphic features & - & - & - & - & $t^{n}$ & $+^{\circ}$ & $t^{p}$ & $+^{9}$ & $4 / 8$ \\
\hline Scoliosis & - & + & - & + & + & - & - & + & $4 / 8$ \\
\hline Small hands/feet & + & - & + & + & - & - & - & + & $4 / 8$ \\
\hline Peripheral neuropathyr & + & + & ND & ND & + & ND & ND & ND & \\
\hline
\end{tabular}

ABR, auditory brainstem response hearing testing; AFP, $\alpha$-fetoprotein; $C T$, computed tomography; $d$., died; DTRs, deep tendon reflexes; EEG, electroencephalography; F, female; IUGR, intrauterine growth restriction; M, male; MRI, magnetic resonance imaging; MRS, magnetic resonance spectroscopy; ND, not determined; NGLY1, N-glycanase 1 .

${ }^{a}$ Normal (MRI 8 months), prominent perivascular spaces and periatrial white matter gliosis (MRI 15 months). bSlightly dilated lateral ventricles (MRI 4 months). 'Mild global reduction in cerebral hemisphere volume and thin corpus callosum and anterior commissure (MRI 17 months), MRS normal. dDelayed myelination (MRI 6 months), delayed myelination, supratentorial atrophy considered secondary to steroid therapy (MRI 10 months), delayed myelination, recovery from supratentorial atrophy (MRI 19 months). eMild ventriculomegaly, small pons and midbrain (CT 10 months), normal (MRI 2 and 4 years). ${ }^{f}$ Prominent lateral ventricles (CT 9 months). ${ }^{~}$ Subtle increased T2 signal in periventricular white matter (MRI 9 years). hInformation on birth head circumference was not available for Patient 2 , and microcephaly was present at birth for Patient 8 , but for all others microcephaly was acquired. 'Blood lactate levels are as follows (normal $<2.2 \mathrm{mmol} / \mathrm{l}$ ); Patient 2: $3.9 \mathrm{mmol} / \mathrm{l}$ at $4 \mathrm{months}$ and $2.7 \mathrm{mmol} / \mathrm{l}$ at 1 year; Patient 3: 3.7-7.5 mmol/l in infancy, $6.4 \mathrm{mmol} / \mathrm{l}$ at 21 months, $6.7 \mathrm{mmol} / \mathrm{l}$ at 31 months, 3.7 at 32 months, and normal values at 19 months, 26 months, 30 months and 3.5 years; Patient 4: $3.2 \mathrm{mmol} / \mathrm{l}$ at 10 months and 15 months, with normal levels at 11 months, 13 months, and 16 months; Patient 7: $2.7 \mathrm{mmol} / \mathrm{l}$ at $7 \mathrm{months}, 2.4-3.2 \mathrm{mmol} / \mathrm{l}$ at 8 months, and $2.4 \mathrm{mmol} / \mathrm{l}$ at 13 months. Blood lactate was mildly elevated on two occasions, thought to be secondary to difficult blood draws, but repeat testing was

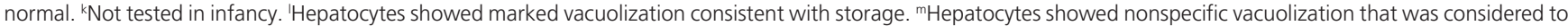
be secondary to agonal hypoxia/ischemia, but such findings may also be consistent with early storage. "Mild ptosis, hypoplastic supraorbital ridges, epicanthic folds, long eyelashes, short nose, thick alveolar ridges, high-arched palate, and mild micrognathia. ${ }^{\circ}$ Epicanthic folds and short nose. ${ }^{\mathrm{p} F r o n t a l}$ bossing, metopic ridge, epicanthic folds, tented upper lip, hypoplastic left nipple, tapered fingers, and mild fourth- and fifth-toe clinodactyly. 'Long eyelashes, arched eyebrows, decreased facial subcutaneous fat, mild proptosis, broad nasal bridge, and crowded dentition. 'As determined by nerve conduction velocity testing. 


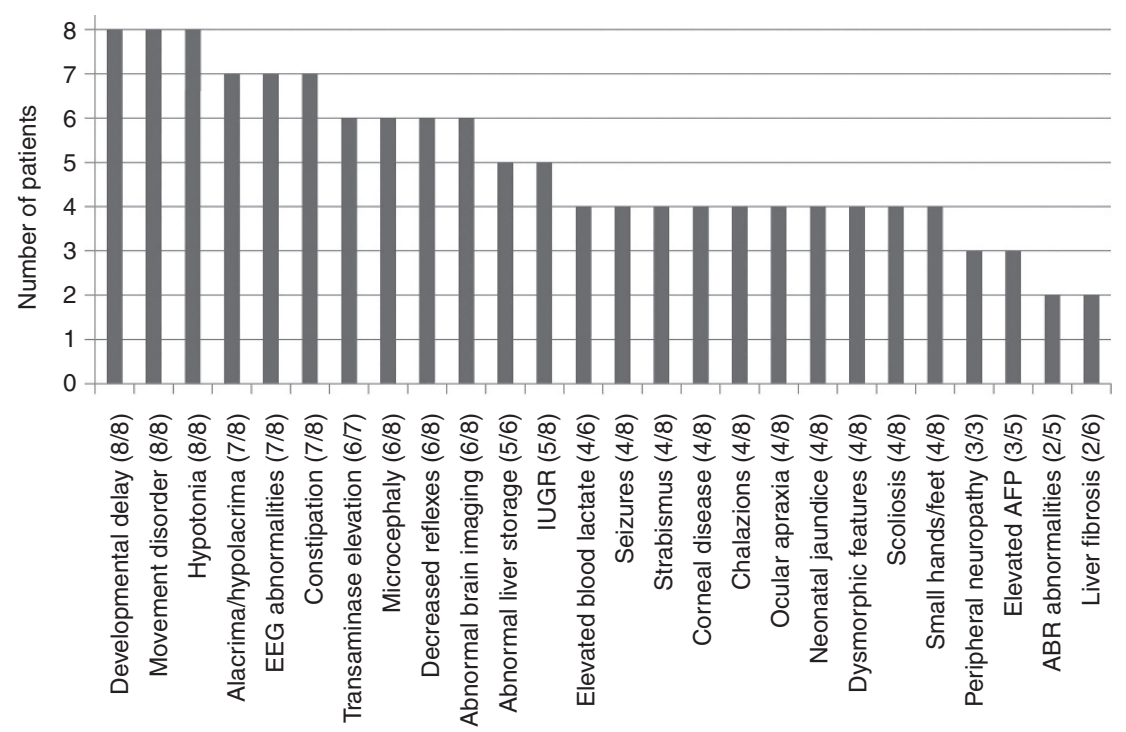

Figure 1 Relative frequency of clinical findings in N-glycanase 1 (NGLY1) deficiency. ABR, auditory brainstem response hearing testing; AFP, $\alpha$-fetoprotein; IUGR, intrauterine growth restriction.

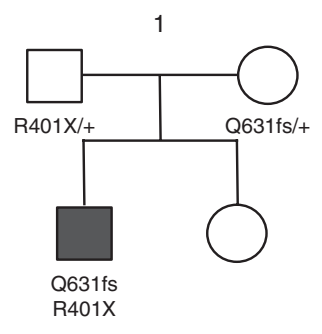

4

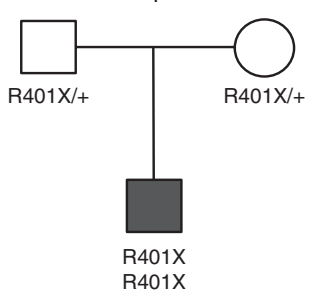

2

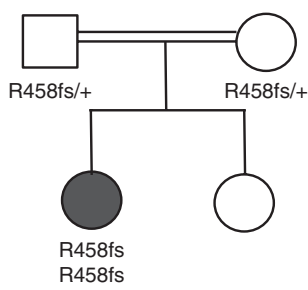

5,6

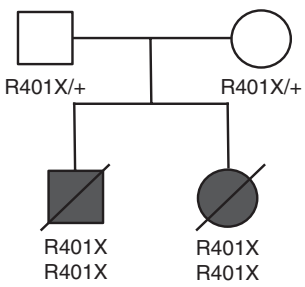

3

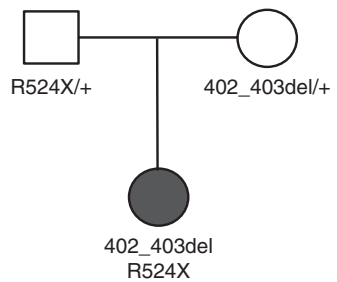

7,8

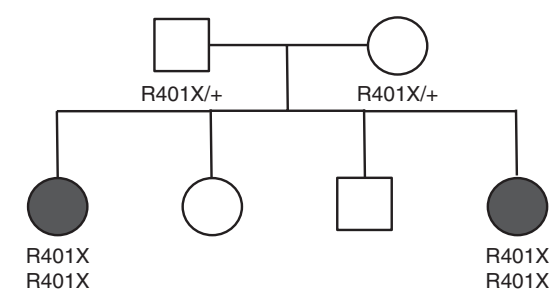

Figure 2 Pedigrees of N-glycanase 1 (NGLY1)-deficient patients.

transaminases and transient thrombocytopenia. In infancy, she was noted to have global developmental delay, acquired microcephaly, bilateral exotropia, hypotonia, and constipation. At $~ 1$ year of age, the parents noticed that she did not make tears while crying, although she had adequate tear production to keep her eyes moist. She also developed staring spells, lasting up to $15 \mathrm{~s}$, at $\sim 1$ year of age; these episodes occur about once daily and can be interrupted by gentle contact. By 17 months of age, she had developed an extrapyramidal movement disorder associated with asynchronous myoclonic jerks of the limbs and shoulders and subtle choreoathetotic movements of the hands and fingers. At 4 years of age, she is able to ambulate unassisted, although her gait is unsteady, and she communicates with the use of vocalizations, gestures, and an assistive communication device.
Patient 4, a now 2-year-old boy, was delivered by Cesarean section at 38 weeks of gestation, when fetal distress was noted on cardiotocography. Intermittent head flexion was noted at 6 months, and an EEG at 8 months showed generalized polyspike discharges. Soon thereafter mild myoclonic seizures with head and body flexion started to occur and evolved to single, symmetric spasms with bilateral arm extension. The epilepsy has been intractable despite many therapeutic trials. Involuntary movements of the upper extremities were also noted at this time. In addition, global developmental delay, abnormal tear production, unilateral ptosis, strabismus, elevated liver transaminases (three to four times upper limit of normal), and constipation were noted in infancy. He has had abnormal tear production, recurrent episodes of keratoconjunctivitis and poor lid closure during sleep, with resultant corneal scarring. His sleep pattern 
is abnormal and is characterized by paucity of deep sleep and frequent awakenings. He has learned to crawl and communicates with vocalizations.

Patient 5, a boy who died at 5 years of age, was born at term following a pregnancy that was complicated by a positive second-trimester serum screening for trisomy 18 and SmithLemli-Opitz syndrome (AFP 1.97 MoM, uE3 0.24 MoM, and human chorionic gonadotrophin $0.48 \mathrm{MoM}$ ). Karyotype on amniocentesis was normal, and measurement of 7-dehydrocholesterol in amniotic fluid excluded Smith-Lemli-Opitz syndrome. He had global developmental delay and constant movements of his arms and legs since early infancy and he developed head bobbing at 7 months. At 8 months, liver transaminase elevations ( 1.5 times the upper limit of normal) were noted, and the elevations persisted until 3.5 years of age. Although his reflexes appeared normal in infancy, they were diminished by 2 years of age and by 38 months could no longer be elicited. During the second year of life, he was noted to have dry eyes that were treated with lubricant drops at bedtime, and microcephaly was present by 16 months. At 2.5 years of age, he developed myoclonic seizures that became intractable despite numerous therapeutic trials. Between the ages of 10 months and 5 years, he showed slow developmental progress but regressed during the last year. He died at age 5 years following a viral illness and a prolonged seizure.

Patient 6 is the younger sister of patient 5 . The pregnancy was also complicated by a positive second-trimester serum screen for trisomy 18 and Smith-Lemli-Opitz syndrome (AFP 0.87 MoM, uE3 0.31 MoM, and human chorionic gonadotrophin $0.57 \mathrm{MoM})$. Karyotype on amniocentesis was normal. She was delivered by cesarean section at 35 weeks of gestation after an ultrasound that was concerning for IUGR was performed. She had jaundice, which required phototherapy, but the neonatal period was otherwise uneventful. By 9 months of age, developmental delay was apparent and she had developed hypotonia, microcephaly, a mildly myopathic-appearing face, and constant involuntary movements, with a tendency to hyperextend her arms and close her fists. At 9.5 months of age, she died unexpectedly in her sleep, and the cause of death remains unknown.

Patient 7, a now 3-year-old girl, was born at term via scheduled Cesarean section and, other than mild jaundice, did not have any neonatal complications. Prenatal screen showed an increased risk for trisomy 21. In infancy, she was noted to have strabismus, hypotonia, athetoid arm and hand movements, clasped hands, and elevated liver transaminases (approximately three times the upper limit of normal). Microcephaly was present by 2 years of age. In addition, severe developmental delay was present, but she has shown slow progress without regression. She learned to sit alone and started to crawl at 2 years of age but has not yet started to communicate with words.

Patient 8, a now 16-year-old girl, is the older sister of Subject 7. Anal stenosis requiring dilatation was noted in the first week of life, but she did not have further neonatal problems and was discharged home on Day 7. In early infancy she was noted to have hypotonia, a movement disorder consisting of head bobbing and extremity athetosis, and developmental delay. At 3 years of age, she could stand unsupported, ambulate with a walker, and started to babble. Seizures developed at 11 years of age, and she lost all mobility. Other medical history includes acquired microcephaly, an inability to completely close her eyes during sleep, chronic conjunctivitis, corneal clouding, hypolacrima, strabismus, hearing impairment, gastro-esophageal reflux, chronic constipation, severe scoliosis, and talipes equinovarus.

\section{Sequencing results}

The nonsense mutation c.1201A $>\mathrm{T}$ (p.R401X) was the most common deleterious allele identified, present in the homozygous state in five of eight cases and in the compound heterozygous state in one case. Patients 2 and 3 did not carry the c.1201A $>$ T (p.R401X) mutation, and their clinical phenotype was relatively mild by comparison (Table 1). Further details related to sequencing are available in the Supplementary Materials and Methods online.

For Patient 1, WES, performed as part of a research protocol, detected putative truncating mutations forming a compound heterozygote genotype in the NGLY1 gene. A maternal frameshift mutation, p.Q631fs, was identified. At the complementary DNA level this is a c.1891delC in transcript ENST00000280700 in exon 12 of NGLY1. The paternal allele was found to be 3_25750426_A, which causes a nonsense mutation, p.R401X, in transcript ENST00000280700. At the complementary DNA level this is c.1201A $>\mathrm{T}$ in exon 8 of NGLY1. ${ }^{15}$

For Patient 2, WES (Baylor College of Medicine Whole Genome Laboratory) revealed a homozygous mutation in exon 9 of the NGLY1 gene denoted as c.1370dupG (p.R458fs). Both parents were confirmed to be heterozygous carriers by Sanger sequencing. The mutation causes a frameshift in codon 458 , causing insertion of 13 incorrect residues before a stop codon is introduced toward the end of exon 9.

For Patient 3, WES and whole-genome sequencing were performed using research protocols available at Baylor College of Medicine and Stanford University. A stop-gain mutation caused by a G>A mutation at position 3:25761670 (hg19) resulting in p.R542X was identified in both the father and the daughter. In addition, a 3-base pair in-frame deletion TTC $>$ beginning at position 3:25775416 (hg19) was identified in both the mother and the daughter (p.402del). An additional G>T variant resulting in a heterozygous single-nucleotide polymorphism at position 3:25777564 was identified in the daughter, mother, and father.

Patients 4-8 were found to be homozygous for the c.1201A $>$ T/p.R401X mutation.

\section{DISCUSSION}

The finding of compound heterozygous loss of function mutations in NGLY1 in the original case suggested that NGLY1 deficiency (OMIM 615273) represents a new disorder ${ }^{15}$ Soon after the initial report, we were able to identify the seven additional cases reported herein, therefore confirming NGLY1 deficiency 
as an inherited disorder of the ERAD pathway and the first to be identified that involves the cytosolic proteasome. The rapid ascertainment of cases occurred over a period of several months and was assisted by strong advocacy from families and connections made possible by the Internet and social media (see accompanying Commentary by Might and Wilsey in this issue). NGLY1-deficient patients have a striking clinical triad, consisting of abnormal tear production, choreoathetosis, and liver disease. In addition, global developmental delay, acquired microcephaly, hypotonia, EEG abnormalities with or without overt seizures, brain imaging abnormalities, a peripheral neuropathy, constipation, and a history of IUGR were common findings. Some patients were noted to have dysmorphic features, but overall these were not considered to be particularly prominent (Table 1). Interestingly, low uE3 was present in three patients, including in two children who died and were found to have significant adrenal cortex vacuolation. Although adrenal function was not specifically evaluated in our patients, some degree of dysfunction remains possible. Further research will be required in order to determine if low $\mathrm{uE} 3$ might provide a clue to underlying NGLY1 deficiency.

The differential diagnosis of inherited conditions featuring alacrima and neurological dysfunction is limited, and we did not identify any other disorder associated with both alacrima and choreoathetosis. Triple-A syndrome (OMIM 231550) is an autosomal recessive condition characterized by adrenocorticotropin hormone-resistant adrenal insufficiency, achalasia, and alacrima. In addition, affected individuals have late-onset cerebellar ataxia, peripheral neuropathy, and mild dementia. ${ }^{16,17}$ Aladin, the gene product of triple-A syndrome, is a member of the WD-repeat family of regulatory proteins, with ubiquitous expression in human tissues but with particularly abundant expression in the cerebellum, adrenal and pituitary glands, and gastrointestinal structures. ${ }^{17}$ Alacrima, achalasia, and mental retardation syndrome (OMIM 615510) is another autosomal recessive condition that features alacrima and neurological involvement, but adrenal function is normal. Alacrima, achalasia, and mental retardation syndrome is caused by mutations in GMPPA, a gene that encodes guanosine diphosphate-mannose pyrophosphorylase A (GMPPA). GMPPA appears to be a regulatory subunit to GMPPB, an enzyme that catalyzes the formation of guanosine diphosphate-mannose. ${ }^{18}$ In addition to the primary clinical findings delineated in its name, alacrima, achalasia, and mental retardation syndrome patients have variable hypotonia, ataxia, spasticity, and hearing impairment. ${ }^{18}$ Alacrima has also been described in different forms of hereditary sensory and autonomic neuropathy, including type III (also known as familial dysautonomia or Riley-Day syndrome) (OMIM 223900) and type VI (OMIM 614653)..$^{19,20}$ Autonomic nervous system dysfunction has been implicated in the alacrima encountered in the above disorders, but the cause of alacrima in NGLY1 deficiency, as well as any possible pathogenic relationship to these other conditions, remains to be elucidated.

The constellation of features present in our cohort is in some ways reminiscent of the clinical features present in the CDGs. ${ }^{21}$
CDGs feature multisystem disease involving in particular the central nervous system, heart, liver, gastrointestinal tract, and endocrine system. Indeed, the possibility of an underlying CDG was high in the differential diagnosis for all cases. On the basis of the initial report of a single NGLY1-deficient patient, a recent CDG review considered that NGLY1 deficiency may even be considered to be the first "congenital disorder of deglycosylation."22 Although there are clear similarities between NGLY1 deficiency and the CDGs as a whole, such as IUGR, failure to thrive, global developmental delay, and liver impairment, these features are nonspecific. Unlike CDGs, NGLY1 deficiency does not appear to be associated with cerebellar atrophy, lipodystrophy, or significant heart manifestations. At this early point of delineation of the clinical phenotypic spectrum, the combination of hypolacrima or alacrima and a movement disorder consisting of tremulousness and varying degrees of choreoathetosis appear to be pathognomonic for NGLY1 deficiency but are not particularly associated with CDGs. In addition, conditions commonly considered in the differential diagnosis included mitochondrial disorders, Rett syndrome and related conditions, and disorders of neurotransmitter metabolism.

Recently, disorders associated with components of the ERAD pathway involving the Golgi apparatus, ER lipid raft-associated protein 1/2 (erlin1/2) complex, and E3-ubiquitin ligase have been identified. These conditions serve as interesting comparisons to deficiency of the cytosolic NGLY1. Mutations in $M A N 1 B 1$, the gene coding for $\alpha-1,2$-mannosidase, are associated with nonsyndromic autosomal recessive intellectual disability and subtle dysmorphic features. ${ }^{23} \alpha-1,2$-Mannosidase is a type II transmembrane protein that is primarily localized to the Golgi apparatus, where it undergoes O-glycosylation and participates in glycoprotein quality control. ${ }^{24,25}$ Profound intellectual disability, developmental regression, and multiple contractures are features associated with autosomal recessive mutations in ERLIN2. In this case, abnormal erlin2 causes impaired ERAD of activated inositol 1,4,5-triphosphate $\left(\mathrm{IP}_{3}\right)$ receptors and other substrates by compromising the structure of the erlin $1 / 2 \mathrm{com}$ plex. ${ }^{26,27}$ Despite the severity of the intellectual disability and neuromuscular findings, the results of brain imaging, electromyography and muscle biopsy appeared normal in the initial erlin2-deficient patients. ${ }^{26}$ Another family was found to have a homozygous null mutation in ERLIN2, with affected individuals presenting with a hereditary spastic paraplegia phenotype..$^{28}$

$\mathrm{IP}_{3}$ receptors form calcium channels in ER membranes and play an important role in mammalian cell signaling. Ultimately, $\mathrm{IP}_{3}$ receptors undergo rapid degradation via the ubiquitin-proteasome pathway in response to cell stimulation. Evidence suggests that RNF170, an E3-ubiquitin ligase, mediates ubiquitination and processing of the $\mathrm{IP}_{3}$ receptor via interaction involving the erlin1/2 complex. ${ }^{27,29}$ Mutations in RNF170 cause a late-onset autosomal dominant sensory ataxia characterized by distal sensory loss, diminished to absent reflexes, wide-based gait, and normal brain imaging. ${ }^{30-32}$ Abnormal E3-ubiquitin ligase activity has also been postulated to play a role in the pathogenesis of an autosomal dominant form of Charcot-Marie-Tooth disease. ${ }^{33}$ 
In short, different steps of the quality control system for targeting and degrading misfolded proteins have been implicated in a number of neurological conditions with varying severity and either autosomal recessive or dominant inheritance.

NGLY1 is a cytoplasmic enzyme that participates in the proteasomal degradation of aberrant glycoproteins that are synthesized in the ER and subsequently translocated to the cytoplasm. ${ }^{1,10,34,35}$ By performing the initial cleavage of bulky N-glycan chains on misfolded glycoproteins, NGLY1 makes further proteolysis possible; glycoproteins thus trimmed can enter the cylinder of the $20 \mathrm{~S}$ proteasome to be acted upon by proteases. ${ }^{34}$ Therefore, NGLY1 deficiency would be expected to result in the accumulation of intact glycoproteins in the cytoplasm. The accumulation of an amorphous substance in the liver of three patients, as well as the vacuolization consistent with storage in two others, is supportive of this role of NGLY1 and may help explain the liver disease noted in our patients. The undefined stored substance likely represents accumulation of misfolded glycoproteins in the cytoplasm that have been retrotranslocated from the ER but cannot undergo further processing. Transferrin isoelectric focusing or mass spectrometry studies in NGLY1-deficient patients have been normal or only subtly abnormal. This is not particularly surprising because these methods detect the absence of or structural alterations in $\mathrm{N}$-glycan chains, ${ }^{36}$ and such abnormalities would not be expected to occur in NGLY1 deficiency. Rather, we would predict that there would be an accumulation of nondegraded cytoplasmic glycoproteins with a relatively normal glycosylation pattern.

The presence in the brains of axonal loss and gliosis suggestive of hypoxic-ischemic encephalopathy suggests that NGLY1 plays a role in maintaining central nervous system integrity. A study in Caenorhabditis elegans detected peripheral nervous system defects, including aberrant neuronal branching, in animals with loss-of-function mutations in png-1, the ortholog of NGLY $1 .{ }^{37}$ Neuronal branching abnormalities possibly relate to the peripheral neuropathy that seems to be relatively common in NGLY1 deficiency, but further studies are needed to determine the underlying pathogenesis of both central and peripheral nervous system abnormalities found in our patients.

The nonsense mutation c.1201A $>\mathrm{T}$ (p.R401X) was the most commonly detected deleterious allele in NGLY1, present in a homozygous state in five of eight cases (Patients 4-8) and in a compound heterozygous state in one case (Patient 1). Clearly, homozygous mutations associated with p.R401X cause a severe phenotype. However, the range of outcomes is variable, with some patients having an early demise and others living at least into their teenage years. Patients 2 and 3 appear to have a relatively mild phenotype and are the only two individuals in the current cohort who do not carry the p.R401X mutation. Aside from these broad considerations, more detailed genotype-phenotype correlations will be possible only with the detection of further patients. Consistent with the severe presentation of NGLY1 deficiency, NGLY1 is among the $20 \%$ of genes in the human genome that are the most intolerant of functional genetic variation in the human population, ${ }^{38}$ and we may expect that less extreme mutations than those seen here may also be associated with disease.

The reasons for the predominance of the p.R401X mutation are unclear. The mutation was observed in only 2 out of 8,598 chromosomes of European ancestry reported in the Exome Variant Server resource. At this carrier frequency, we would expect one affected individual among approximately every 20 million people due to homozygosity for this mutation. The predominance of this mutation among cases could reflect the relative rarity of other null and hypomorphic mutations in the gene, and/or an increased severity of cases due to p.R401X mutations, leading to a higher chance of ascertainment. Consistent with these possibilities, collectively, apparent null mutations are extremely rare in NGLY1 in humans. It is also noteworthy that all patients carrying p.R401X mutations are of European ancestry, suggesting the possibility of a founder mutation in that community. Interestingly, there is one p.R401X mutation observed among African-American chromosomes in the Exome Variant Server. Whether this reflects the presence of the mutation in populations of African ancestry or stems from European chromosomes among African Americans is unclear. In future work, it will be important to determine whether all p.R401X mutations occur on a common haplotype background and to characterize the geographic pattern of functional variation in NGLY1.

In summary, NGLY1 deficiency is characterized by a constellation of unique features that should enable clinical recognition of this condition. The more salient features are elevation of AFP and liver enzymes in infancy with relative normalization in early childhood, accumulation of a substance with staining properties similar to glycogen (presumably misfolded glycoproteins) in hepatocyte cytoplasm, absent tears resulting in blepharitis and corneal ulceration, a movement disorder, and peripheral neuropathy. However, the transient nature of the AFP and liver transaminase elevations may make older or more mildly affected individuals difficult to detect; therefore, affected older children and adults may remain undiagnosed. However, the widespread clinical availability of WES and whole-genome sequencing will likely result in further cases being detected, including those with a more mild phenotype.

\section{SUPPLEMENTARY MATERIAL}

Supplementary material is linked to the online version of the paper at http://www.nature.com/gim

\section{ACKNOWLEDGMENTS}

The authors gratefully acknowledge the generosity of the parents of all patients in providing samples and clinical data for this study. The Bertrand Might Research Fund (H.H.F.) provided support for the study of subjects 1 and 2. Stephen T. Warren, PhD, provided support for sequencing of subjects 7 and 8 . Michael and Ellen Michelson, Tom and Diane Might, and Bobbie and Mike Wilsey provided additional support for this research. C.P.S. is a recipient of a Clinical Scientist Development Award by the Doris Duke Charitable Foundation. His work is generously supported by the Joan and Stanford Alexander Family. The sequencing work 
for subjects 5 and 6 was carried out through a research project (FORGE Canada) funded by the government of Canada through Genome Canada, the Canadian Institutes of Health Research, and the Ontario Genomics Institute. The FORGE Canada Steering Committee includes Kym Boycott (University of Ottawa), Jan Friedman (University of British Columbia, Jacques Michaud (Université de Montréal), Francois Bernier (University of Calgary), Michael Brudno (University of Toronto), Bridget Fernandez (Memorial University), Bartha Knoppers (McGill University), Mark Samuels (Université de Montréal), and Steve Scherer (University of Toronto). Support from grant number U54-HG003273 to R.A.G.

\section{DISCLOSURE}

The authors declare no conflict of interest.

\section{REFERENCES}

1. Takahashi N. Demonstration of a new amidase acting on glycopeptides. Biochem Biophys Res Commun 1977;76:1194-1201.

2. Plummer TH Jr, Elder JH, Alexander S, Phelan AW, Tarentino AL. Demonstration of peptide: $\mathrm{N}$-glycosidase $\mathrm{F}$ activity in endo-beta- $\mathrm{N}$-acetylglucosaminidase $\mathrm{F}$ preparations. J Biol Chem 1984;259:10700-10704.

3. Suzuki T, Park H, Hollingsworth NM, Sternglanz R, Lennarz WJ. PNG1, a yeast gene encoding a highly conserved peptide: $\mathrm{N}$-glycanase. J Cell Biol 2000;149:1039-1052.

4. Xin F, Wang S, Song L, Liang Q, Qi Q. Molecular identification and characterization of peptide: N-glycanase from Schizosaccharomyces pombe. Biochem Biophys Res Commun 2008;368:907-912.

5. Masahara-Negishi Y, Hosomi A, Della Mea M, Serafini-Fracassini D, Suzuki T. A plant peptide: $\mathrm{N}$-glycanase orthologue facilitates glycoprotein ER-associated degradation in yeast. Biochim Biophys Acta 2012;1820:1457-1462.

6. Gosain A, Lohia R, Shrivastava A, Saran S. Identification and characterization of peptide: N-glycanase from Dictyostelium discoideum. BMC Biochem 2012;13:9.

7. Seko A, Kitajima $K$, Inoue $Y$, Inoue S. Peptide: $N$-glycosidase activity found in the early embryos of Oryzias latipes (Medaka fish). The first demonstration of the occurrence of peptide: $\mathrm{N}$-glycosidase in animal cells and its implication for the presence of a de-N-glycosylation system in living organisms. J Biol Chem 1991;266:22110-22114.

8. Suzuki T, Seko A, Kitajima K, Inoue Y, Inoue S. Identification of peptide:Nglycanase activity in mammalian-derived cultured cells. Biochem Biophys Res Commun 1993:194:1124-1130.

9. Suzuki T, Seko A, Kitajima K, Inoue $Y$, Inoue S. Purification and enzymatic properties of peptide: $\mathrm{N}$-glycanase from $\mathrm{C} 3 \mathrm{H}$ mouse-derived L-929 fibroblast cells. Possible widespread occurrence of post-translational remodification of proteins by N-deglycosylation. J Biol Chem 1994;269:17611-17618.

10. Park H, Suzuki T, Lennarz WJ. Identification of proteins that interact with mammalian peptide: $\mathrm{N}$-glycanase and implicate this hydrolase in the proteasome-dependent pathway for protein degradation. Proc Natl Acad Sci USA 2001;98:11163-11168.

11. Jarosch $E$, Taxis $C$, Volkwein $C$, et al. Protein dislocation from the ER requires polyubiquitination and the AAA-ATPase Cdc48. Nat Cell Bio/ 2002;4:134-139.

12. Kato K, Kamiya Y. Structural views of glycoprotein-fate determination in cells. Glycobiology 2007;17:1031-1044.

13. Kamiya Y, Satoh T, Kato K. Molecular and structural basis for N-glycandependent determination of glycoprotein fates in cells. Biochim Biophys Acta 2012;1820:1327-1337

14. Kamiya $Y$, Uekusa $Y$, Sumiyoshi A, et al. NMR characterization of the interaction between the PUB domain of peptide: $\mathrm{N}$-glycanase and ubiquitin-like domain of HR23. FEBS Lett 2012;586:1141-1146.

15. Need AC, Shashi V, Hitomi Y, et al. Clinical application of exome sequencing in undiagnosed genetic conditions. J Med Genet 2012;49:353-361.
16. Tullio-Pelet A, Salomon R, Hadj-Rabia S, et al. Mutant WD-repeat protein in triple-A syndrome. Nat Genet 2000;26:332-335.

17. Handschug K, Sperling S, Yoon SJ, Hennig S, Clark AJ, Huebner A. Triple A syndrome is caused by mutations in AAAS, a new WD-repeat protein gene. Hum Mol Genet 2001;10:283-290.

18. Koehler K, Malik M, Mahmood S, et al. Mutations in GMPPA cause a glycosylation disorder characterized by intellectual disability and autonomic dysfunction. Am J Hum Genet 2013;93:727-734.

19. Anderson SL, Coli R, Daly IW, et al. Familial dysautonomia is caused by mutations of the IKAP gene. Am J Hum Genet 2001;68:753-758.

20. Edvardson $S$, Cinnamon $Y$, Jalas $C$, et al. Hereditary sensory autonomic neuropathy caused by a mutation in dystonin. Ann Neurol 2012;71:569572.

21. Freeze HH, Eklund EA, Ng BG, Patterson MC. Neurology of inherited glycosylation disorders. Lancet Neurol 2012;11:453-466.

22. Freeze $\mathrm{HH}$. Understanding human glycosylation disorders: biochemistry leads the charge. J Biol Chem 2013;288:6936-6945.

23. Rafiq MA, Kuss AW, Puettmann L, et al. Mutations in the alpha 1,2-mannosidase gene, MAN1B1, cause autosomal-recessive intellectual disability. Am J Hum Genet 2011;89:176-182.

24. Pan S, Wang S, Utama B, et al. Golgi localization of ERManl defines spatial separation of the mammalian glycoprotein quality control system. Mol Biol Cell 2011;22:2810-2822

25. Pan S, Cheng X, Sifers RN. Golgi-situated endoplasmic reticulum $\alpha-1$, 2-mannosidase contributes to the retrieval of ERAD substrates through a direct interaction with $\gamma$-COP. Mol Biol Cell 2013;24:1111-1121.

26. Yildirim $Y$, Orhan EK, Iseri SA, et al. A frameshift mutation of ERLIN2 in recessive intellectual disability, motor dysfunction and multiple joint contractures. Hum Mol Genet 2011;20:1886-1892.

27. Pearce MM, Wormer DB, Wilkens S, Wojcikiewicz RJ. An endoplasmic reticulum (ER) membrane complex composed of SPFH1 and SPFH2 mediates the ER-associated degradation of inositol 1,4,5-trisphosphate receptors. J Biol Chem 2009:284:10433-10445.

28. Alazami AM, Adly N, Al Dhalaan H, Alkuraya FS. A nullimorphic ERLIN2 mutation defines a complicated hereditary spastic paraplegia locus (SPG 18). Neurogenetics 2011;12:333-336.

29. Lu JP, Wang Y, Sliter DA, Pearce MM, Wojcikiewicz RJ. RNF170 protein, an endoplasmic reticulum membrane ubiquitin ligase, mediates inosito 1,4,5-trisphosphate receptor ubiquitination and degradation. J Biol Chem 2011;286:24426-24433.

30. Valdmanis PN, Simões Lopes AA, Gros-Louis F, Stewart JD, Rouleau GA, Dupré $N$. A novel neurodegenerative disease characterised by posterior column ataxia and pyramidal tract involvement maps to chromosome 8p12-8q12.1. J Med Genet 2004;41:634-639.

31. Valdmanis PN, Brunet D, St-Onge J, Weston L, Rouleau GA, Dupré N. A founder haplotype for autosomal dominant sensory ataxia in Eastern Canada. Neurology 2006;67:2239-2242.

32. Valdmanis PN, Dupré $N$, Lachance $M$, et al. A mutation in the RNF170 gene causes autosomal dominant sensory ataxia. Brain 2011;134(Pt 2):602-607.

33. Saifi GM, Szigeti K, Wiszniewski W, et al. SIMPLE mutations in CharcotMarie-Tooth disease and the potential role of its protein product in protein degradation. Hum Mutat 2005;25:372-383.

34. Tanabe K, Lennarz WJ, Suzuki T. A cytoplasmic peptide: N-glycanase. Meth Enzymol 2006:415:46-55

35. Suzuki T, Park H, Lennarz WJ. Cytoplasmic peptide:N-glycanase (PNGase) in eukaryotic cells: occurrence, primary structure, and potential functions. FASEB $J$ 2002;16:635-641.

36. Freeze $\mathrm{HH}$, Aebi M. Altered glycan structures: the molecular basis of congenital disorders of glycosylation. Curr Opin Struct Bio/ 2005;15:490-498.

37. Habibi-Babadi N, Su A, de Carvalho CE, Colavita A. The N-glycanase png-1 acts to limit axon branching during organ formation in Caenorhabditis elegans. J Neurosci 2010;30:1766-1776.

38. Petrovski S, Wang Q, Heinzen EL, Allen AS, Goldstein DB. Genic intolerance to functional variation and the interpretation of personal genomes. PLoS Genet 2013;9:e1003709. 